\title{
SEPARATIVE CANCELLATION FOR PROJECTIVE MODULES OVER EXCHANGE RINGS
}

by

P. ARA, K.R. GOODEARL, K.C. O'MEARA and E. PARDO

No. 142

May, 1996

\begin{abstract}
A separative ring is one whose finitely generated projective modules satisfy the property $A \oplus A \simeq A \oplus B \cong B \oplus B \Longrightarrow A \sim B$. This condition is shown to provide a key to a number of outstanding cancellation problems for finitely generated projective modules over exchange rings. It is shown that the class of separate exchange rings is very broad, and, notably, closed under extensions of ideals by factor rings. That is, if an exchange ring $R$ has an ideal $I$ with $I$ and $R / I$ both separative, then $R$ is separative.
\end{abstract}




\title{
SEPARATIVE CANCELLATION FOR PROJECTIVE MODULES OVER EXCHANGE RINGS
}

\author{
P. Ara, K.R. Goodearl, K.C. O'Meara and E. Pardo
}

\begin{abstract}
A separative ring is one whose finitely generated projective modules satisfy the property $A \oplus A \cong A \oplus B \cong B \oplus B \Longrightarrow A \cong B$. This condition is shown to provide a key to a number of outstanding cancellation problems for finitely generated projective modules over exchange rings. It is shown that the class of separative exchange rings is very broad, and, notably, closed under extensions of ideals by factor rings. That is, if an exchange ring $R$ has an ideal $I$ with $I$ and $R / I$ both separative, then $R$ is separative.
\end{abstract}

\section{INTRODUCTION}

In order to study the direct sum decomposition theory of a class of modules, it is important to know how close the class is to having an 'ideal' decomposition theory. Of course in the presence of suitable chain conditions, each module in the class is a direct sum of indecomposable modules, and an ideal decomposition theory would yield uniqueness of decompositions into indecomposables, as in the Krull-Remak-Schmidt-Azumaya Theorem. However, when the class of modules is not built from indecomposables, an 'ideal' decomposition theory must be formulated in terms of different conditions. Among the most basic and useful are:

(C) Cancellation: $A \oplus C \cong B \oplus C \Longrightarrow A \cong B$.

(UR) Uniqueness of $n$-th roots: $\bigoplus_{i=1}^{n} A \cong \bigoplus_{i=1}^{n} B \Longrightarrow A \cong B$.

These conditions have been studied in many contexts. We focus on the class $F P(R)$ of finitely generated projective modules over a (von Neumann) regular ring $R$, or, more generally, an exchange ring. It follows from a combination of results of Fuchs, Kaplansky and Handelman that the regular rings whose finitely generated projective modules satisfy (C) are precisely those with stable rank one (cf. [25, Theorem 4.5 and Proposition 4.12]). This result was recently extended to exchange rings by Yu [50, Theorem 9]. However, the second author has constructed simple regular rings with stable rank one over which (UR) fails $[\mathbf{2 7}]$. On the other hand, right self-injective rings $R$ constitute a nice class of exchange rings such that $F P(R)$ satisfies uniqueness of $n$-th roots for all $n$ (cf. [24]), but in general $F P(R)$ does not satisfy cancellation.

1991 Mathematics Subject Classification. 16D40, 16D70, 16E50, 46L05, 06F05, $20 \mathrm{M} 14$.

Key words and phrases. Separative ring, exchange ring, von Neumann regular ring, $\mathrm{C}^{*}$-algebra, real rank zero, refinement monoid, separative monoid, stable rank, extension.

The research of the first and fourth authors was partially supported by a grant from the DGICYT (Spain) and by the Comissionat per Universitats i Recerca de la Generalitat de Catalunya. That of the second author was partially supported by a grant from the NSF (USA). 
We say that $R$ is a separative ring if the following condition holds for all $A, B \in F P(R)$ :

$$
A \oplus A \cong A \oplus B \cong B \oplus B \Longrightarrow A \cong B
$$

Obviously the class of separative rings includes all rings $R$ such that $F P(R)$ satisfies either cancellation or uniqueness of $n$-th roots. As we will prove, it includes many more - perhaps all - exchange rings. One important source of construction of separative exchange rings is provided by our Extension Theorem for separative exchange rings (Theorem 4.2). It states that, for an exchange ring $R$ with a (two-sided) ideal $I$, the ring $R$ is separative if and only if $I$ and $R / I$ are separative. (Here, saying that $I$ is separative is equivalent to saying that all the unital rings $e R e$ are separative for $e=e^{2} \in I$.) This is in sharp contrast with the class of exchange rings with stable rank one (see for example [25, Example 4.26]).

We also prove that separativity for an exchange ring $R$ drastically reduces the possible values of the stable rank of $R$, to 1,2 , or $\infty$. It is conceivable that all exchange rings are separative. As we show, this would imply affirmative answers to five outstanding open questions in the theory of regular rings (see Section 6). This illustrates the role of separativity as a unifying principle for cancellation problems over exchange rings.

The term separativity is borrowed from semigroup theory. Following Clifford and Preston $[17, \mathrm{p} .131]$, an abelian monoid $M$ is said to be separative if for all $a, b \in M$,

$$
a+a=a+b=b+b \quad \Longrightarrow \quad a=b .
$$

They chose this term because, by a 1956 result of Hewitt and Zuckerman [32], $M$ is separative if and only if the characters of $M$ separate elements of $M$. (See [17, Theorem 5.59]. For this result, a character of $M$ can be any semigroup homomorphism of $M$ into the multiplicative semigroup of complex numbers.) We have chosen our terminology in such a way that a ring $R$ is separative if and only if the monoid $V(R)$ of isomorphism classes of finitely generated projective $R$-modules is a separative monoid. We have found it useful to apply semigroup methods in $V(R)$ to prove some of our results.

In the last section, we give some applications of our results to the field of operator algebras. Since $\mathrm{C}^{*}$-algebras with real rank zero are exchange rings (Theorem 7.2), our results can be applied to this important class of $\mathrm{C}^{*}$-algebras. Moreover, this theorem shows that the exchange property provides a uniform algebraic viewpoint for direct sum decomposition properties over regular rings and $\mathrm{C}^{*}$-algebras with real rank zero, and hence it gives further motivation to work within the class of exchange rings.

Here is a brief outline of the paper. In Section 1, we recall some basic definitions and we prove some preparatory lemmas. In Section 2, we develop some basic characterizations and initial applications of separativity. Section 3 is devoted to the study of stable rank conditions on exchange rings. In particular, it is proved that the only possible values of the stable rank of a separative exchange ring are 1, 2, or $\infty$. We prove in Section 4 one of the main results of this paper, namely the Extension Theorem for separative exchange rings. Section 5 gives a corresponding extension result for the smaller class of strongly separative exchange rings, which is obtained as a corollary of the above. Finally, Sections 6 and 7 examine some particular features of our results for the important classes of regular rings and $\mathrm{C}^{*}$-algebras with real rank zero, respectively. 
Since most of the literature on regular rings and exchange rings is written for the unital case, we shall operate under the dictum "all rings have units" for most of the paper. When discussing $\mathrm{C}^{*}$-algebras in the final section, however, we state our results for not necessarily unital algebras as far as possible. Our notation is standard; see for instance $[\mathbf{9}, \mathbf{2 5}]$. In particular, we write $n A$ for the direct sum of $n$ copies of a module $A$. We use the notation $A \lesssim B$ to indicate that a module $A$ is isomorphic to a direct summand of a module $B$.

All monoids considered in this paper will be abelian monoids, written additively.

\section{EXCHANGE RINGS AND REFINEMENT MONOIDS}

We begin by recalling some basic concepts that are central to our work, in particular the notions of 'exchange ring' and 'refinement monoid', and we introduce a natural refinement monoid $V(R)$ that faithfully records direct sum decompositions of finitely generated projective modules over any exchange ring $R$.

An $R$-module $M$ has the exchange property (see [19]) if for every $R$-module $A$ and any decompositions

$$
A=M^{\prime} \oplus N=\bigoplus_{i \in I} A_{i}
$$

with $M^{\prime} \cong M$, there exist submodules $A_{i}^{\prime} \subseteq A_{i}$ such that

$$
A=M^{\prime} \oplus\left(\bigoplus_{i \in I} A_{i}^{\prime}\right)
$$

(It follows from the modular law that $A_{i}^{\prime}$ must be a direct summand of $A_{i}$ for all $i$.) If the above condition is satisfied whenever the index set is finite, $M$ is said to satisfy the finite exchange property. Clearly a finitely generated module satisfies the exchange property if and only if it satisfies the finite exchange property. It should be emphasized that the direct sums in the definition of the exchange property are internal direct sums of submodules of $A$. One advantage of the resulting internal direct sum decompositions (as opposed to isomorphisms with external direct sums) rests on the fact that direct summands with common complements are isomorphic - e.g., $N \cong \bigoplus_{i \in I} A_{i}^{\prime}$ above since each of these summands of $A$ has $M^{\prime}$ as a complementary summand.

Following Warfield [45], we say that a ring $R$ is an exchange ring if $R_{R}$ satisfies the (finite) exchange property. By [45, Corollary 2], this definition is left-right symmetric. If $R$ is an exchange ring, then every finitely generated projective $R$-module has the exchange property (by [19, Lemma 3.10], the exchange property passes to finite direct sums and to direct summands), and so the endomorphism ring of any such module is an exchange ring. Further, idempotents lift modulo all ideals of an exchange ring [39, Theorem 2.1, Corollary 1.3].

The class of exchange rings is quite large. It includes all semiregular rings (i.e., rings which modulo the Jacobson radical are regular and have idempotent-lifting), all $\pi$-regular rings, and more; see $[45,43]$. Further, all $\mathrm{C}^{*}$-algebras with real rank zero are exchange rings, as we prove in Section 7.

The following criterion for exchange rings was obtained independently by Nicholson and the second author. 
Lemma 1.1. [39, Theorem $2.1 ; 29$, p. 167] Let $R$ be a ring. Then, $R$ is an exchange ring if and only if for every element $a \in R$ there exists an idempotent $e \in R$ such that $e \in R a$ and $1-e \in R(1-a)$.

For any ring $R$ we denote by $F P(R)$ the class of finitely generated projective right $R$-modules. The following common refinement property for direct sums in $F P(R)$ is well. known over regular rings [25, Theorem 2.8].

Proposition 1.2. Assume that $R$ is an exchange ring and that $A_{1}, A_{2}, B_{1}, B_{2} \in F P(R)$. If $A_{1} \oplus A_{2} \cong B_{1} \oplus B_{2}$, there exist decompositions $A_{i}=A_{i 1} \oplus A_{i 2}$ for $i=1,2$ such that $A_{1 j} \oplus A_{2 j} \cong B_{j}$ for $j=1,2$.

Proof. This is a special case of [19, Theorem 4.1]. We give the easy proof for the reader's convenience. It suffices to prove the existence of common refinements for any internal direct sum decomposition $P=A \oplus B=C \oplus D$, where $P, A, B, C, D \in F P(R)$. Now $A$ has the exchange property. Then $P=A \oplus C^{\prime} \oplus D^{\prime}$ for some submodules $C^{\prime} \subseteq C$ and $D^{\prime} \subseteq D$; moreover, $C=C^{\prime} \oplus C^{\prime \prime}$ and $D=D^{\prime} \oplus D^{\prime \prime}$ for some $C^{\prime \prime}, D^{\prime \prime} \in F P(R)$. Now $P=A \oplus B=$ $A \oplus C^{\prime} \oplus D^{\prime}$, whence $B \cong C^{\prime} \oplus D^{\prime}$. Also, $P=C^{\prime} \oplus D^{\prime} \oplus\left(C^{\prime \prime} \oplus D^{\prime \prime}\right)=\left(C^{\prime} \oplus D^{\prime}\right) \oplus A$, and thus $A \cong C^{\prime \prime} \oplus D^{\prime \prime}$.

The above common refinement property is fundamental to almost all work on direct sum decompositions of finitely generated projective modules over an exchange ring $R$. (See, e.g., [25] for its use in the case of a regular ring.) Since this property involves only isomorphisms and direct sums, it can be expressed in the monoid of isomorphism classes of objects from $F P(R)$. This provides a convenient notational shorthand that simplifies many proofs. Furthermore, the monoid viewpoint provides a perspective which is sometimes more suggestive than a module-theoretic viewpoint.

For any ring $R$, we denote by $V(R)$ the monoid of isomorphism classes of objects from $F P(R)$. We shall use square brackets to denote these isomorphism classes; hence, the addition operation in $V(R)$ is given by $[P]+[Q]=[P \oplus Q]$. This monoid can also be described as the monoid of equivalence classes of idempotents from $\bigcup_{n=1}^{\infty} M_{n}(R)$. In particular, this shows the right-left symmetry of $V(R)$.

A monoid $M$ is said to be a refinement monoid (e.g., [21], [47]) if whenever $a+b=c+d$ in $M$, there exist $x, y, z, t \in M$ such that $a=x+y$ and $b=z+t$ while $c=x+z$ and $d=y+t$. It is sometimes convenient to record such simultaneous refinements in the format of a refinement matrix

$$
\begin{aligned}
& c d \\
& a\left(\begin{array}{ll}
x & y \\
z & t
\end{array}\right)
\end{aligned}
$$

(This notation means that the sum of each row equals the element labelling that row, and similarly for column sums.) By induction, the refinement property also holds for sums with more than two terms, i.e., given $a_{1}+\cdots+a_{m}=b_{1}+\cdots+b_{n}$ in $M$, there exist elements $x_{i j} \in M$ (for $i=1, \ldots, m$ and $j=1, \ldots, n$ ) such that each $a_{i}=x_{i 1}+\cdots+x_{i n}$ and each $b_{j}=x_{1 j}+\cdots+x_{m j}$. Refinement monoids have been extensively studied in recent years; see for example [21], [40], [47], [48]. The class of refinement monoids is very large, as can be seen from the following result: Every abelian semigroup can be embedded in a refinement monoid [30, Theorem 1; 21, Theorem 5.1]. 
Corollary 1.3. If $R$ is an exchange ring, then $V(R)$ is a refinement monoid.

Proof. This is just a restatement of Proposition 1.2.

This result should be contrasted with the fact that $K_{0}^{+}$of an exchange ring does not always have the refinement property [38].

We will make use of a few standard concepts from the theory of abelian monoids. For instance, we will occasionally assume that our monoids are conical, meaning that elements $x, y$ can satisfy $x+y=0$ only when $x=y=0$. Note that the monoids $V(R)$ are always conical, since a direct sum of modules is zero only when the summands are zero.

Let $M$ be a monoid. For $x, y \in M$ we will write $x \leq y$ if there exists $z \in M$ such that $y=x+z$. This translation-invariant preorder (it is reflexive and transitive, but not necessarily antisymmetric) is called the algebraic preorder in $M[\mathbf{1 0}, 2.1 .1]$. It is sometimes useful to assume that $M$ has an order-unit, i.e., an element $u \in M$ such that every element of $M$ is bounded above by a positive multiple of $u$. In the monoid $V(R)$, we have $[A] \leq[B]$ if and only if $A$ is isomorphic to a direct summand of $B$. Note that $[R]$ is an order-unit in $V(R)$; more generally, a class $[A] \in V(R)$ is an order-unit precisely when $A$ is a generator in the category of $R$-modules.

Finally, we need a concept of 'ideal' for monoids that corresponds, when applied to $V(R)$, to ideals of the ring $R$. The appropriate concept is not that of ideal as used in semigroup theory, but rather an analog of the 'o-ideals' studied in the theory of partially ordered groups (cf. [23, p. 20]).

An $o$-ideal of a monoid $M$ is a submonoid $S$ of $M$ such that $S$ is hereditary with respect to the algebraic ordering, i.e., $y \leq x$ for $y \in M$ and $x \in S$ implies $y \in S$. (Equivalently, a nonempty subset $S$ of $M$ is an o-ideal if and only if we have $a+b \in S \Longleftrightarrow a, b \in S$ for $a, b \in M$.) Observe that the set of invertible elements of $M$ (i.e., its group of units) is an o-ideal of $M$, contained in every o-ideal. The monoid $M$ is said to be o-simple provided $M$ is not a group and the only ideals of $M$ are $M$ and the group of units. In particular, a nonzero conical monoid is o-simple if and only if all its nonzero elements are order-units.

Given an o-ideal $S$ of $M$; we define a congruence $\sim_{S}$ on $M$ by setting $a \sim_{S} b$ if and only if there exist $e, f \in S$ such that $a+e=b+f$. Note that $a \sim_{S} 0$ if and only if $a \in S$. Let $M / S$ be the factor monoid obtained from the congruence $\sim_{S}$. We shall write elements of $M / S$ in the form $[a]_{S}$. In case $M$ is a refinement monoid, the congruence $\sim_{S}$ can be expressed in the following alternate way: $a \sim_{S} b$ if and only if there exist $c \in M$ and $g, h \in S$ such that $a=c+g$ and $b=c+h$.

Let $R$ be a ring and $I$ a (two-sided) ideal of $R$. Denote by $F P(I)$ the set of projectives $P \in F P(R)$ such that $P=P I$, and by $V(I)$ the set of isomorphism classes $[P] \in V(R)$ for $P \in F P(I)$. If $R$ is an exchange ring, then every finitely generated projective $R$-module is isomorphic to a finite direct sum of principal right ideals of $R$ generated by idempotents, so that $V(I)$ is the submonoid of $V(R)$ generated by $\left\{[e R] \mid e=e^{2} \in I\right\}$.

If $R$ is an exchange ring, then so is $R / I$ for every ideal $I$ of $R$. The next result determines $V(R / I)$ as a quotient of $V(R)$.

Proposition 1.4. Let $R$ be an exchange ring and $I$ an ideal of $R$. Then $V(I)$ is an o-ideal of $V(R)$ and $V(R) / V(I) \cong V(R / I)$.

Proof. It is clear that $V(I)$ is an o-ideal of $V(R)$. The tensor product functor $(-) \otimes_{R}(R / I)$ induces a natural homomorphism $\phi: V(R) \rightarrow V(R / I)$, and $\phi$ in turn induces a natural 
homomorphism $\psi: V(R) / V(I) \rightarrow V(R / I)$. First, notice that as $R$ is an exchange ring, idempotents lift modulo $I$, whence $\phi$ and $\psi$ are surjective.

To prove that $\psi$ is injective, it suffices to show that whenever $A, B \in F P(R)$ with $A / A I \cong B / B I$, there exist decompositions $A=A_{1} \oplus A_{2}$ and $B=B_{1} \oplus B_{2}$ such that $A_{1} \cong B_{1}$ while $A_{2}=A_{2} I$ and $B_{2}=B_{2} I$. This amounts to a problem about idempotent matrices over $R$ which become equivalent modulo $I$. Since all matrix rings over $R$ are exchange rings, it is enough to solve the $1 \times 1$ case. Therefore we may assume, without loss of generality, that $A=e R$ and $B=f R$ for some idempotents $e, f \in R$.

Now $e R / e I \cong f R / f I$, and so there exist $x \in e R f$ and $y \in f R e$ such that $x y \equiv e$ $(\bmod I)$ and $y x \equiv f(\bmod I)$. Observe that $x y \in e R e$. Since $e R e$ is an exchange ring with unit $e$, there exists an idempotent $g \in x y R e$ such that $e-g \in(e-x y) R e$; then $e-g \in I$. Write $g=x y t$ with $t \in e R g$, and observe that $e \equiv g \equiv e t=t(\bmod I)$. On the other hand, the element $h=y t x \in f R f$ is an idempotent such that $g \sim h$ and $f \equiv y x=y e x \equiv y t x=h$ $(\bmod I)$. Therefore $A=g R \oplus(e-g) R$ and $B=h R \oplus(f-h) R$ with $g R \cong h R$ while $(e-g) R=(e-g) R I$ and $(f-h) R=(f-h) R I$, as desired.

Although it is not needed in the present paper, we mention that for any exchange ring $R$, the lattice of ideals of $V(R)$ is isomorphic to the lattice of semiprimitive ideals of $R$ [40, Teorema 4.1.7].

We conclude this section with some further observations about ideals that will be needed later.

Lemma 1.5. Let $R$ be an exchange ring and $I$ an ideal of $R$.

(a) Given any idempotents $e_{1}, \ldots, e_{n} \in I$, there exists an idempotent $e \in I$ such that $e_{1}, \ldots, e_{n} \in R e R$.

(b) $V(I)$ equals a directed union of o-ideals $V(R e R)$ where $e$ runs through the idempotents in $I$.

(c) $V(R e R) \cong V(e R e)$ for any idempotent $e \in R$.

Proof. (a) It suffices to consider the case $n=2$. Since $e_{1} R \oplus\left(1-e_{1}\right) R=e_{2} R \oplus\left(1-e_{2}\right) R$, Proposition 1.2 yields a decomposition $e_{2} R=A \oplus B$ such that $A$ and $B$ are isomorphic to direct summands of $e_{1} R$ and $\left(1-e_{1}\right) R$ respectively. Hence, there exist idempotents $f \in e_{1} R e_{1}$ and $f^{\prime} \in\left(1-e_{1}\right) R\left(1-e_{1}\right)$ such that $\left(f+f^{\prime}\right) R \cong e_{2} R$. Note that $f^{\prime} \in R e_{2} R \subseteq I$. Thus $e:=e_{1}+f^{\prime}$ is an idempotent in $I$, and obviously $e_{1}=e e_{1} \in R e R$. On the other hand, $f+f^{\prime}=e\left(f+f^{\prime}\right) \in R e R$, and therefore $e_{2} \in R\left(f+f^{\prime}\right) R \subseteq R e R$.

(b) This is clear from (a).

(c) Since the additive functor $(-) \otimes_{e} R_{e} e R$ sends $F P(e R e)$ into $F P(R e R)$, it induces a monoid homomorphism $\phi: V(e R e) \rightarrow V(R e R)$. The functor $(-) \otimes_{R} R e$, on the other hand, does not send all projective $R$-modules to projective $e R e$-modules. Consider a projective $A \in F P(R e R)$. Since $A$ is finitely generated, $A=a_{1} e R+\cdots+a_{n} e R$ for some $a_{i}$, whence there exists an epimorphism $n(e R) \rightarrow A$, and so $n(e R) \cong A \oplus B$ for some $R$-module $B$. Consequently, $n(e R e) \cong A e \oplus B e$, and hence $A e \in F P(e R e)$. Therefore $(-) \otimes_{R} R e$ induces a monoid homomorphism $\psi: V(R e R) \rightarrow V(e R e)$.

It is clear that $\psi \phi$ is the identity on $V(e R e)$. Observe that for all right $R$-modules $A$ there is a natural homomorphism $\eta_{A}: A \otimes_{R} R e \otimes_{e} e e R \rightarrow A$ given by multiplication, and that $\eta_{e R}$ is an isomorphism. If $A \in F P(R e R)$, then as above $A$ is isomorphic to a direct 
summand of $n(e R)$ for some $n$, whence $\eta_{A}$ is an isomorphism. Therefore $\phi \psi$ is the identity on $V(R e R)$.

\section{Separativity}

We develop some basic characterizations and initial applications of separativity in this section. Let us say that a class $\mathcal{C}$ of modules is separative if for all $A, B \in \mathcal{C}$ we have

$$
A \oplus A \cong A \oplus B \cong B \oplus B \quad \Longrightarrow \quad A \cong B
$$

A ring $R$ will be called a separative ring if $F P(R)$ is a separative class of modules. This is clearly more general than rings for which $F P(R)$ is cancellative. We give some concrete classes of examples later, after developing some equivalent formulations of separativity.

Since some of our work with separative exchange rings $R$ involves calculations with the monoids $V(R)$, we turn next to separativity for monoids. The monoid context is also convenient for demonstrating the equivalence of various forms of this condition. Recall that a monoid $M$ is separative if for all $a, b \in M$,

$$
a+a=a+b=b+b \quad \Longrightarrow \quad a=b .
$$

Note that our terminology has been chosen so that a ring $R$ is separative precisely when the monoid $V(R)$ is separative. In describing alternate forms of this condition, it is convenient to use the following notation, borrowed from [48, Section 2]. For $a, b \in M$ we write $a \propto b$ if there exists a positive integer $n$ such that $a \leq n b$; equivalently, $a$ belongs to the o-ideal generated by $b$.

Since every semigroup can be embedded in a refinement monoid [30, Theorem $1 ; 21$, Theorem 5.1], there exist non-separative refinement monoids. In fact, every o-simple conical monoid can be embedded in an o-simple conical refinement monoid [48, Corollary 2.7], and so there exist non-separative o-simple refinement monoids. The first example of such a monoid was constructed by Bergman [8].

Lemma 2.1. Given a monoid $M$, the following conditions are equivalent:

(i) $M$ is separative.

(ii) For $a, b \in M$, if $2 a=2 b$ and $3 a=3 b$, then $a=b$.

(iii) For $a, b \in M$, if there exists $n \in \mathbb{N}$ such that $n a=n b$ and $(n+1) a=(n+1) b$, then $a=b$.

(iv) For $a, b, c \in M$, if $a+c=b+c$ with $c \propto a$ and $c \propto b$, then $a=b$.

In case $M$ is a refinement monoid, separativity is also equivalent to the following:

(v) For $a, b, c \in M$, if $a+2 c=b+2 c$, then $a+c=b+c$.

Proof. The equivalence of (i) and (iv) amounts to Hewitt and Zuckerman's result that $M$ is separative if and only if its archimedean components are cancellative [32, Corollary 4.15.1] (cf. [17, Theorem 4.16]). Our approach via condition (iii) gives an alternate proof. The implication (iii) $\Longrightarrow$ (iv) is based on an argument of Kimura and Tsai [34, Theorem 1] (cf. [10, Theorem 2.1.9]).

(i) $\Longrightarrow$ (ii). Observe that $2(2 a)=2(a+b)=2 a+(a+b)$. Then by (i), we have $2 a=a+b$. Since $2 a=2 b$ also, we conclude using (i) again that $a=b$. 
(ii) $\Longrightarrow$ (iii). If $n \in \mathbb{N}$ such that $n a=n b$ and $(n+1) a=(n+1) b$, then $n a+a=n a+b$. It follows that $n a+k a=n a+k b=n b+k b$ for all $k \in \mathbb{N}$. If $n>1$, then $2 n-2 \geq n$ and so $2(n-1) a=2(n-1) b$ and $3(n-1) a=3(n-1) b$. We conclude using (ii) that $(n-1) a=(n-1) b$. Therefore by induction on $n$, we obtain $a=b$.

(iii) $\Longrightarrow$ (iv). Assume that $a+c=b+c$ with $c \leq k a$ and $c \leq k b$ for some $k \in \mathbb{N}$. Write $k a=c+d$ for some $d \in M$. We have

$$
(k+1) a=a+c+d=b+c+d=k a+b .
$$

Then $(k+2) a=(k+1) a+b=k a+2 b$, and so on: $(k+r) a=k a+r b$ for all $r \in \mathbb{N}$. By symmetry, $(k+r) b=k b+r a$ for all $r \in \mathbb{N}$. In particular, taking $r=k$ we obtain $2 k a=k a+k b=2 k b$. Further, $(2 k+1) a=k a+(k+1) a=2 k a+b=(2 k+1) b$, and therefore $a=b$ using (iii).

(iv) $\Longrightarrow$ (i). Obvious.

Now assume that $M$ is a refinement monoid. The implication (iv) $\Longrightarrow(v)$ is clear. For the converse, consider elements $a, b, c \in M$ such that $a+c=b+c$ while $c \propto a$ and $c \propto b$. Since $c \leq k a$ for some $k \in \mathbb{N}$, we have $c=c_{1}+\cdots+c_{k}$ for some $c_{i} \leq a$. It suffices to cancel the $c_{i}$ successively from the equality $a+c_{1}+\cdots+c_{k}=b+c_{1}+\cdots+c_{k}$, and so there is no loss of generality in assuming that $c \leq a$. Similarly, we may reduce to the case that $c \leq b$. Now write $a=a^{\prime}+c$ and $b=b^{\prime}+c$ for some $a^{\prime}, b^{\prime} \in M$. Then $a^{\prime}+2 c=b^{\prime}+2 c$ and so $a^{\prime}+c=b^{\prime}+c$ by (v), that is, $a=b$. This shows that (v) $\Longrightarrow$ (iv).

Lemma 2.1 gives characterizations of separativity (using isomorphism in place of equality) for any class $\mathcal{C}$ of modules which is closed under finite direct sums - simply form the monoid of isomorphism classes. (To avoid set-theoretical difficulties, one can apply the lemma to monoids of isomorphism classes of modules taken from subsets of $\mathcal{C}$.) In particular, (ii) shows that separativity of $\mathcal{C}$ occurs precisely when 'multiple-isomorphism' $(n A \cong n B$ for all $n>1$ ) coincides with isomorphism. In this light, it appears that 'multiple-isomorphism' within the class of finite rank torsionfree abelian groups is a considerably finer equivalence relation than 'near-isomorphism', since by [46, Theorem 5.9] the latter is equivalent to $n A \cong n B$ for some $n$.

Our main interest in Lemma 2.1 is its application to the monoids $V(R)$. Thus, separativity for a ring $R$ is equivalent to any of the following conditions holding for all modules $A, B, C \in F P(R)$ :

(ii) If $2 A \cong 2 B$ and $3 A \cong 3 B$, then $A \cong B$.

(iii) If there exists $n \in \mathbb{N}$ such that $n A \cong n B$ and $(n+1) A \cong(n+1) B$, then $A \cong B$.

(iv) If $A \oplus C \cong B \oplus C$ and $C$ is isomorphic to direct summands of both $m A$ and $n B$ for some $m, n \in \mathbb{N}$, then $A \cong B$.

We refer to property (iv) as separative cancellation. In case $R$ is an exchange ring, separativity is also equivalent to the condition

(v) If $A \oplus 2 C \cong B \oplus 2 C$, then $A \oplus C \cong B \oplus C$

for $A, B, C \in F P(R)$. In [4, Theorem 3.4], we show that $R$ is separative if and only if all regular square matrices over each corner ring $e R e$ are diagonalizable over $e R e$.

Many large classes of rings of interest are separative. For instance:

(1) All rings $R$ with stable rank 1 , since $F P(R)$ is cancellative in that case $[22$, Theorem 2]. This includes all unit-regular rings as well as all strongly $\pi$-regular rings [3, Theorem 4], and hence all algebraic algebras over a field. 
(2) Any ring whose finitely generated projective modules enjoy uniqueness of square roots $(2 A \cong 2 B \Longrightarrow A \cong B$ ), because of condition (ii) above. This includes all right $\aleph_{0}$-continuous regular rings $[1$, Theorem 2.13] and all right self-injective rings (e.g., [24, Theorem 3]), as well as all $\mathrm{AW}^{*}$-algebras - even all Rickart $\mathrm{C}^{*}$-algebras (see [2, Theorem 2.7]).

(3) In light of the Extension Theorem that we prove in Section 4, many seemingly pathological examples of regular rings in the literature, from Bergman's example of a directly finite regular ring which is not unit-regular [25, Example 4.26] to the rings constructed in [5] and [6], are actually separative.

The examples just mentioned illustrate the point that all known classes of exchange rings are separative. Outside the class of exchange rings, however, separativity can easily fail. Examples include the first Weyl algebra and the coordinate ring of the 2-sphere (cf. [28, Section 2]). It is not difficult to see that a commutative ring $R$ is separative only if $F P(R)$ is actually cancellative.

Proposition 2.2. The class of separative exchange rings is closed under taking corners, finite matrix rings, arbitrary direct products, direct limits, and factor rings.

Proof. Closure under direct products and direct limits is easy, using Lemma 1.1 and the definition of separativity. We leave that part of the proof to the reader. That separativity passes to factor rings of exchange rings is easiest to prove using monoid calculations. Since we will need the corresponding monoid result later, we defer the proof to Lemma 4.3. Finally, let $R$ be an exchange ring and $T$ either a corner $e R e$ or a matrix $\operatorname{ring} M_{n}(R)$. Then $T$ is an exchange ring because it is the endomorphism ring of an object in $F P(R)$. In the first case, $V(T) \cong V(R e R) \subseteq V(R)$ by Lemma 1.5, while in the second case $V(T)=V\left(T e_{11} T\right) \cong V\left(e_{11} T e_{11}\right) \cong V(R)$ by the same lemma, where $e_{11}$ is the usual matrix unit. In either case, separativity therefore passes from $V(R)$ to $V(T)$.

Our first application of separativity is to the stability of direct finiteness under the formation of matrix rings. Recall that a module $A$ is called directly finite or directly infinite according to whether or not $A$ is isomorphic to a proper direct summand of itself. A ring $R$ is said to be directly finite provided $R_{R}$ is a directly finite module; equivalently, $x y=1$ implies $y x=1$ for $x, y \in R$. We say that $R$ is stably finite if all matrix rings $M_{n}(R)$ are directly finite; equivalently, if all finitely generated projective $R$-modules are directly finite.

Proposition 2.3. Any directly finite separative ring $R$ is stably finite.

Proof. Suppose that $n R \oplus C \cong n R$ for some $n \in \mathbb{N}$ and $C \in F P(R)$. Then we have $(n-1) R \oplus(R \oplus C) \cong(n-1) R \oplus R$. Since $R$ is separative, we can cancel $(n-1) R$ from both sides, obtaining $R \oplus C \cong R$. Then since $R$ is directly finite, we conclude that $C=0$. Therefore $R$ is stably finite.

An interesting situation in which separativity occurs is the case of an o-simple "purely infinite' monoid, as follows. This is a monoid version of an argument of Cuntz [20, Theorem 1.4, Proposition 1.5].

Proposition 2.4. Let $M$ be an o-simple conical monoid, and assume that for every nonzero element $a \in M$, there exists a nonzero element $b \in M$ such that $a+b=a$. Then $M$ is 
a separative refinement monoid. In fact, the set $M^{*}=M \backslash\{0\}$ is a group.

Proof. Since $M$ is conical, $M^{*}$ is closed under addition. We claim that given any $x, y \in M^{*}$, there exists an element $z \in M^{*}$ such that $x+z=y$. By hypothesis, $y+b=y$ for some $b \in M^{*}$, and we observe that $y+n b=y$ for all $n \in \mathbb{N}$. Since $M$ is o-simple, $x \leq n b$ for some $n$. Then $x+x^{\prime}=n b$ for some $x^{\prime} \in M$, and $x+\left(x^{\prime}+y\right)=y$. Since $M$ is conical, $x^{\prime}+y \in M^{*}$, and the claim is proved.

The claim above implies that $M^{*}$ is a group (e.g., [18, Section 3.2, Theorem 1]). In particular, $M^{*}$ is cancellative, and it follows immediately that $M$ is separative. It also follows easily that $M^{*}$ is a refinement monoid, and therefore that $M$ is one as well.

Corollary 2.5. Let $R$ be a simple ring. If every nonzero finitely generated projective $R$-module is directly infinite, then $R$ is separative.

Corollary 2.6. If $R$ is a simple exchange ring which is not separative, then $R$ has a corner $e R e$ which is a directly finite, simple, non-separative exchange ring.

Proof. By Corollary 2.5, there must be some nonzero $A \in F P(R)$ which is directly finite. Now $A=A_{1} \oplus \cdots \oplus A_{n}$ for some $A_{i}$ which are isomorphic to direct summands of $R_{R}$, and these $A_{i}$ must be directly finite. Hence, there exists a nonzero idempotent $e \in R$ such that $e R$ is a directly finite module. Thus $e R e$ is a directly finite simple exchange ring. Since $V(e R e) \cong V(R e R)=V(R)$ by Lemma 1.5, eRe cannot be separative.

Although separativity for a ring $R$ is an 'external' condition in that it involves all the modules from $F P(R)$, it is equivalent to a corresponding 'internal' version involving direct summands of $R$ in case $R$ is an exchange ring (Corollary 2.9). En route to proving this, we give the main reduction step as a lemma that will be used again later.

Lemma 2.7. Let $M$ be a refinement monoid, and let $a, b, c \in M$ with $a+c=b+c$.

(i) There exist decompositions $a=a_{1}+a_{2}$ and $b=b_{1}+b_{2}$ together with $c=c_{1}+c_{2}$ in $M$ such that $a_{1}=b_{1}$ and $a_{2}+c_{2}=b_{2}+c_{2}=c$.

(ii) If $c \leq a$ and $c \leq b$, there exist decompositions as in (i) such that $c_{2} \leq a_{2}$ and $c_{2} \leq b_{2}$.

Proof. (i) Since $a+c=b+c$, there exists a refinement matrix

$$
\begin{aligned}
& \text { b c } \\
& \begin{array}{l}
a \\
c
\end{array}\left(\begin{array}{ll}
a_{1} & a_{2} \\
b_{2} & c_{2}
\end{array}\right)
\end{aligned}
$$

Set $b_{1}=a_{1}$ and $c_{1}=a_{2}$.

(ii) We modify the decompositions obtained in (i). Since $c_{2} \leq c \leq a=a_{1}+a_{2}$, we can write $c_{2}=c^{\prime}+c^{\prime \prime}$ with $c^{\prime} \leq a_{1}$ and $c^{\prime \prime} \leq a_{2}$. Then $a_{1}=c^{\prime}+d$ for some $d$, and we obtain decompositions

$$
a=d+\left(a_{2}+c^{\prime}\right), \quad b=d+\left(b_{2}+c^{\prime}\right), \quad c=\left(c_{1}+c^{\prime}\right)+c^{\prime \prime}
$$

such that $\left(a_{2}+c^{\prime}\right)+c^{\prime \prime}=\left(b_{2}+c^{\prime}\right)+c^{\prime \prime}=c$ and $c^{\prime \prime} \leq a_{2} \leq a_{2}+c^{\prime}$. Thus, after replacing the original decompositions of $a, b, c$ with these new ones, we may assume that $c_{2} \leq a_{2}$.

Note that the procedure just performed reduces $c_{2}$ while enlarging $a_{2}$ and $b_{2}$. Therefore we need only repeat the procedure with the roles of $a$ and $b$ reversed. 
Proposition 2.8. Let $M$ be a refinement monoid containing an order-unit $u$. Then $M$ is separative if and only if, for $a, b, c \in M$, if $a+c=b+c \leq u$ with $c \leq a$ and $c \leq b$, then $a=b$.

Proof. Assume that the given special cases of separativity hold, and suppose that $a+c=$ $b+c$ for $a, b, c \in M$ with $c \propto a$ and $c \propto b$. As in the proof of Lemma 2.1[(v) $\Longrightarrow(\mathrm{iv})]$, we can reduce to the case that $c \leq\{u, a, b\}$.

Now there exist decompositions $a=a_{1}+a_{2}, b=b_{1}+b_{2}$, and $c=c_{1}+c_{2}$ as in Lemma 2.7(i). Since $a_{2}+c_{2}=c \leq u$, we may - by hypothesis - cancel $c_{2}$ from the equation $a_{2}+c_{2}=b_{2}+c_{2}$. Therefore $a_{2}=b_{2}$, and hence $a=b$ as desired.

Corollary 2.9. Let $R$ be an exchange ring. Then $R$ is separative if and only if whenever $A \oplus C \cong B \oplus C \lesssim^{\oplus} R$ with $C \lesssim^{\oplus} A$ and $C \lesssim^{\oplus} B$, it follows that $A \cong B$.

\section{STABLE RANK}

It has been known for some time that stable rank conditions on endomorphism rings imply various cancellation properties $[22,46]$. For a regular ring $R$, a combination of results of Kaplansky, Fuchs and Handelman shows that $R$ has stable rank 1 if and only if $R_{R}$ cancels from direct sums (cf. [25, Theorem 4.5 and Proposition 4.12]). This equivalence was recently extended to exchange rings by $\mathrm{Yu}[\mathbf{5 0}$, Theorem 9]; see also [16, Theorem 3]. Further, Menal and Moncasi proved that bounds on the stable rank of a regular ring $R$ are equivalent to cancellation conditions in $F P(R)$ [36, Theorem 3].

We prove that for any exchange ring $R$, the stable rank of $R$ is determined by cancellation conditions within $F P(R)$. This allows us to restrict the stable rank severely in the separative case - namely, the stable rank of a separative exchange ring can only be 1,2 , or $\infty$.

Recall that a ring $R$ satisfies the $n$-stable rank condition (for a given positive integer $n$ ) if whenever $a_{1}, \ldots, a_{n+1} \in R$ with $a_{1} R+\cdots+a_{n+1} R=R$, there exist elements $b_{1}, \ldots, b_{n} \in R$ such that

$$
\left(a_{1}+a_{n+1} b_{1}\right) R+\cdots+\left(a_{n}+a_{n+1} b_{n}\right) R=R .
$$

If $n$ is the least positive integer such that $R$ satisfies the $n$-stable rank condition, then $R$ is said to have stable rank $n$, and we write $\operatorname{sr}(R)=n$. If no such $n$ exists, then $\operatorname{sr}(R)=\infty$. The reader is referred to [44] for the basic properties of stable rank.

Lemma 3.1. [39, Proposition 2.9] The following conditions are equivalent for a projective module $P$ :

(i) $P$ has the finite exchange property.

(ii) If $P=M_{1}+\cdots+M_{n}$, where the $M_{i}$ are submodules of $P$, then there is a decomposition $P=P_{1} \oplus \cdots \oplus P_{n}$ with $P_{i} \subseteq M_{i}$ for each $i$.

(iii) If $P=M+N$, where $M$ and $N$ are submodules of $P$, then there exists a direct summand $P_{1}$ of $P$ such that $P_{1} \subseteq M$ and $P=P_{1}+N$.

Theorem 3.2. Let $R$ be an exchange ring, $P \in F P(R)$, and $n \in \mathbb{N}$. Then $\operatorname{sr}\left(\operatorname{End}_{R}(P)\right) \leq$ $n$ if and only if the following condition holds:

(†) Whenever $X, Y \in F P(R)$ with $n P \oplus X \cong P \oplus Y$, there exists $Q \in F P(R)$ such that $n P \cong P \oplus Q$ and $Y \cong X \oplus Q$. 
Proof. Set $S=\operatorname{End}_{R}(P)$. The implication $(\Longrightarrow)$ is due to Warfield [46, Theorem 1.3], and is valid without the exchange property.

Conversely, assume that $(\dagger)$ holds, and let $a_{1}, \ldots, a_{n+1}$ be elements in $S$ such that $a_{1} S+\cdots+a_{n+1} S=S$. By Lemma 3.1, there exist orthogonal idempotents $e_{1}, \ldots, e_{n+1} \in S$ such that $e_{1}+\cdots+e_{n+1}=1$ and $e_{i} P \subseteq a_{i} P$ for all $i$; it follows that $e_{i} S \subseteq a_{i} S$. Choose elements $x_{i} \in S e_{i}$ such that $e_{i}=a_{i} x_{i}$, and set $f_{i}=x_{i} a_{i}$. Then $a_{i} f_{i}=a_{i} x_{i} a_{i}=e_{i} a_{i}$. Further, $f_{i} x_{i}=x_{i}$, and hence $f_{i}^{2}=f_{i}$.

Note that $e_{i} P \cong f_{i} P$ for all $i$. Hence, we have

$$
\begin{aligned}
n P \oplus e_{n+1} P & =f_{1} P \oplus\left(1-f_{1}\right) P \oplus \cdots \oplus f_{n} P \oplus\left(1-f_{n}\right) P \oplus e_{n+1} P \\
& \cong e_{1} P \oplus \cdots \oplus e_{n+1} P \oplus\left(1-f_{1}\right) P \oplus \cdots \oplus\left(1-f_{n}\right) P \\
& =P \oplus\left(1-f_{1}\right) P \oplus \cdots \oplus\left(1-f_{n}\right) P .
\end{aligned}
$$

By $(\dagger)$, there exists a projective $Q \in F P(R)$ such that $n P \cong P \oplus Q$ and

$$
\left(1-f_{1}\right) P \oplus \cdots \oplus\left(1-f_{n}\right) P \cong e_{n+1} P \oplus Q
$$

Therefore there exist elements $t_{i} \in e_{n+1} S\left(1-f_{i}\right)$ and $s_{i} \in\left(1-f_{i}\right) S e_{n+1}$ such that $\sum_{i=1}^{n} t_{i} s_{i}=e_{n+1}$. Note that $a_{i} s_{i}=a_{i}\left(1-f_{i}\right) s_{i}=\left(1-e_{i}\right) a_{i} s_{i}$ for all $i \leq n$.

For $i=1, \ldots, n$, set $z_{i}=e_{n+1} a_{i}\left(1-f_{i}\right)$ and $c_{i}=x_{n+1}\left(t_{i}-z_{i}\right)$, and observe that

$$
a_{i}+a_{n+1} c_{i}=a_{i}+e_{n+1}\left(t_{i}-z_{i}\right)=a_{i}+t_{i}-z_{i}
$$

and $\left(1-e_{i}-e_{n+1}\right) a_{i} s_{i}=a_{i} s_{i}-z_{i} s_{i}$. Then set

$$
d_{i}=s_{i}+x_{i}-x_{i} \sum_{\substack{j=1 \\ j \neq i}}^{n} a_{j} s_{j}
$$

for $i=1, \ldots, n$. Since $x_{i}=f_{i} x_{i}$ while $t_{i} f_{i}=z_{i} f_{i}=0$, we compute that

$$
\begin{aligned}
\sum_{i=1}^{n}\left(a_{i}+a_{n+1} c_{i}\right) d_{i} & =\sum_{i=1}^{n}\left[\left(a_{i}+t_{i}-z_{i}\right) s_{i}+e_{i}-e_{i} \sum_{\substack{j=1 \\
j \neq i}}^{n} a_{j} s_{j}\right] \\
& =\sum_{i=1}^{n} t_{i} s_{i}+\sum_{i=1}^{n} e_{i}+\sum_{i=1}^{n}\left(a_{i}-z_{i}\right) s_{i}-\sum_{\substack{i, j=1 \\
i \neq j}}^{n} e_{j} a_{i} s_{i} \\
& =1+\sum_{i=1}^{n}\left(a_{i}-z_{i}\right) s_{i}-\sum_{i=1}^{n}\left(1-e_{i}-e_{n+1}\right) a_{i} s_{i}=1 .
\end{aligned}
$$

Therefore $\sum_{i=1}^{n}\left(a_{i}+a_{n+1} c_{i}\right) S=S$, which verifies that $\operatorname{sr}(S) \leq n$.

Theorem 3.2 shows in particular that if $P$ cancels from direct sums in $F P(R)$, then $\operatorname{End}_{R}(P)$ has stable rank 1. The converse follows from Evans' theorem [22, Theorem 2]. Hence, we obtain a new proof of Yu's result that an exchange ring $R$ has stable rank 1 if and only if $R_{R}$ cancels from direct sums [50, Theorem 9]. 
Theorem 3.3. Let $R$ be a separative exchange ring and $P$ a finitely generated projective $R$-module.

(a) $\operatorname{sr}\left(\operatorname{End}_{R}(P)\right)$ can only be 1, 2, or $\infty$.

(b) $\operatorname{sr}\left(\operatorname{End}_{R}(P)\right)<\infty$ if and only if the following condition holds:

$$
2 P \oplus X \cong P \oplus Y \quad \Longrightarrow \quad P \oplus X \cong Y
$$

for all $X, Y \in F P(R)$.

Proof. It is clear from Theorem 3.2 that the condition given in (b) implies $\operatorname{sr}\left(\operatorname{End}_{R}(P)\right) \leq$ 2. It remains to deduce this condition from the assumption that $\operatorname{sr}\left(\operatorname{End}_{R}(P)\right)=n<\infty$. Suppose that $2 P \oplus X \cong P \oplus Y$ for some $X, Y \in F P(R)$; we wish to show that $P \oplus X \cong Y$. Because of the separativity of $F P(R)$, it suffices to prove that $P \delta^{\oplus} n Y$. By adding $(n-1) Y$ to both sides of the isomorphism $2 P \oplus X \cong P \oplus Y$ and repeatedly replacing $P \oplus Y$ by $2 P \oplus X$ on the left hand side, we obtain $n P \oplus(P \oplus n X) \cong P \oplus n Y$. Since $\operatorname{End}_{R}(P)$ has stable rank $n$, there exists $Q \in F P(R)$ such that $n P \cong P \oplus Q$ and $n Y \cong(P \oplus n X) \oplus Q$. Therefore $P \lesssim n Y$ as desired.

Theorem 3.4. Let $R$ be a separative exchange ring. If $R$ is simple and directly finite, then $\operatorname{sr}(R)=1$.

Proof. In view of Theorem 3.2, it suffices to show that $F P(R)$ is cancellative. Suppose $A, B, C$ are in $F P(R)$ with $A \oplus C \cong B \oplus C$. If one of $A$ or $B$ is 0 , then so is the other, since $R$ is stably finite (Proposition 2.3). If both $A$ and $B$ are nonzero, then by simplicity of $R$, we have $C \lesssim n A$ and $C \lesssim n B$ for some $n$. Now by separative cancellation in $F P(R)$, we obtain $A \cong B$. Therefore $F P(R)$ is cancellative, as desired. (An alternative method of proof can be found at the end of [28, Section 3]).

Returning to Theorem 3.2 for a moment, we note that this result shows that the stable rank of an exchange ring $R$ is determined by the monoid $V(R)$. To simplify the connection, it is convenient to introduce a definition of stable rank for elements of a monoid, modelled on the condition appearing in the theorem.

Let $M$ be a monoid, $a$ an element of $M$, and $n \in \mathbb{N}$. We say that $a$ satisfies the $n$-stable rank condition provided the following implication holds: Whenever $n a+x=a+y$ for some $x, y \in M$, there exists $b \in M$ such that $n a=a+b$ and $y=x+b$. (Note that the $n$-stable rank condition implies the $m$-stable rank condition for all integers $m \geq n$.) The stable rank of $a$, denoted $\operatorname{sr}(a)$, is the least positive integer $n$ such that $a$ satisfies the $n$-stable rank condition (if such an $n$ exists), or $\infty$ (if no such $n$ exists).

Theorem 3.2 can now be restated as follows: Given a finitely generated projective module $P$ over an exchange ring $R$, the stable rank of the ring $\operatorname{End}_{R}(P)$ equals the stable rank of the element $[P]$ in the monoid $V(R)$. In particular, $\operatorname{sr}(R)=\operatorname{sr}([R])$.

We conclude the section by noting a recent result of $\mathrm{Wu}$ and Tong: If $R$ is an exchange ring such that all idempotents in $R / J(R)$ are central, then $F P(R)$ is cancellative [49, Theorem 2.5].

\section{EXTENSIONS}

We now develop an Extension Theorem for separativity, which shows that the class of separative exchange rings is closed under extensions in the following sense - whenever $R$ 
is an exchange ring with an ideal $I$ such that $I$ and $R / I$ are both separative, then $R$ is separative. (The exchange property for $R$ must be assumed at the outset, since the class of exchange rings is not closed under extensions.)

We say that an ideal $I$ of a ring $R$ is a separative ideal if $V(I)$ is a separative monoid. The following characterization of separative ideals of exchange rings is clear from Lemma 1.5 .

Lemma 4.1. Let $R$ be an exchange ring and $I$ an ideal of $R$. Then $I$ is separative if and only if all corner rings $e R e$, for idempotents $e \in I$, are separative.

Theorem 4.2. (Extension Theorem) Let $R$ be an exchange ring and $I$ an ideal of $R$. Then $R$ is separative if and only if $I$ and $R / I$ are separative.

Proof. The result will follow from Theorem 4.5 and Proposition 1.4.

Theorem 4.2 shows that separativity leads to better closure properties than cancellativity. Namely, if $R$ is an exchange ring and $I$ is an ideal of $R$ such that $V(R / I)$ and $V(I)$ are cancellative then $V(R)$ need not be cancellative; see for example [25, Example 4.26] or [36, Example 1]. However, Theorem 4.2 shows that $V(R)$ must at least be separative, and we shall see in the next section that it in fact satisfies a rather strong form of separativity.

We derive Theorem 4.2 from a corresponding extension theorem for separative refinement monoids. The monoid approach proved invaluable here. Indeed, we were unable to prove Theorem 4.2 with module-theoretic methods, and it was only the perspective afforded by phrasing the problem in terms of refinement monoids that indicated a route to the solution.

Lemma 4.3. Let $M$ be a separative monoid and $S$ an o-ideal of $M$. Then $M / S$ is separative.

Proof. Assume that $2[a]_{S}=[a]_{S}+[b]_{S}=2[b]_{S}$ for some $a, b \in M$. Then there exist $e_{1}, e_{2}, e_{3} \in S$ such that $2 a+e_{1}=a+b+e_{2}=2 b+e_{3}$. After replacing each $e_{i}$ by $e_{i}+e_{3}$, we may assume in addition that $e_{3} \leq 2 e_{2}$. Now observe that

$$
a+\left(a+e_{1}\right)=a+\left(b+e_{2}\right)
$$

with $a \leq a+e_{1}$ and $a \leq 2 a+e_{1}=2 b+e_{3} \leq 2\left(b+e_{2}\right)$. By Lemma 2.1(iv), we obtain $a+e_{1}=b+e_{2}$ since $M$ is separative. Therefore $[a]_{S}=[b]_{S}$.

Lemma 4.4. Let $M$ be a refinement monoid and $S$ a separative o-ideal of $M$. Assume that $a+e=b+e$ for some $a, b \in M$ and $e \in S$ such that $e \propto a$ and $e \propto b$. Then $a=b$.

Proof. As in the proof of Lemma 2.1[(v) $\Longrightarrow$ (iv)], we can reduce to the case that $e \leq a$ and $e \leq b$. By Lemma 2.7, there exist decompositions

$$
a=a_{1}+a_{2}, \quad b=b_{1}+b_{2}, \quad e=e_{1}+e_{2}
$$

such that $a_{1}=b_{1}$ and $a_{2}+e_{2}=b_{2}+e_{2} \leq e$, while also $e_{2} \leq a_{2}$ and $e_{2} \leq b_{2}$. Since $e$ lies in $S$, so do $a_{2}, b_{2}, e_{2}$. Hence, $a_{2}=b_{2}$ because $S$ is separative, and therefore $a=b$. 
We are now ready to prove our extension theorem for separative refinement monoids. The hypotheses of the theorem include the assumption that the whole monoid has refinement, since, in general, an extension of two separative refinement monoids has neither separativity nor refinement. For example, consider the (abelian) monoid $M$ generated by symbols $a, b, c$ subject to the relation $a+2 c=b+2 c$. The order ideal $S$ of $M$ generated by $c$ is just the free abelian monoid on $c$, and the factor $M / S$ is the free abelian monoid generated by $[a]_{S}=[b]_{S}$. However, $M$ is neither separative $(2(a+c)=(a+c)+(b+c)=2(b+c)$, yet $a+c \neq b+c)$ nor a refinement monoid (the relation $a+2 c=b+2 c$ cannot be refined).

Theorem 4.5. Let $M$ be a refinement monoid and $S$ an o-ideal of $M$. Then $M$ is separative if and only if $S$ and $M / S$ are separative.

Proof. If $M$ is separative, then $S$ is obviously separative and $M / S$ is separative by Lemma 4.3 .

Assume now that $S$ and $M / S$ are separative and that $2 a=a+b=2 b$ for some $a, b \in M$. We have to prove that $a=b$. Let $M^{\prime}$ be the o-ideal generated by $a$, which equals the oideal generated by $b$. Set $S^{\prime}=M^{\prime} \cap S$. Then $S^{\prime}$ is a separative o-ideal of $M^{\prime}$, and $M^{\prime} / S^{\prime}$ is isomorphic to a submonoid of $M / S$, whence $M^{\prime} / S^{\prime}$ is separative. Thus, changing notation, we can assume that $M$ is the o-ideal generated by $a$.

Since $M / S$ is separative, we have $[a]_{S}=[b]_{S}$ and so $a+x=b+y$ for some $x, y \in S$. Now

$$
2 a+x=a+b+x=2 b+y=2 a+y .
$$

Apply refinement to the equality $a+a+x=a+a+y$ to obtain a refinement matrix

$$
\begin{gathered}
a \\
a \\
a
\end{gathered}\left(\begin{array}{ccc}
a_{11} & a_{12} & y_{1} \\
a_{21} & a_{22} & y_{2} \\
x_{1} & x_{2} & y_{3}
\end{array}\right)
$$

Next, apply refinement to the equality $a_{11}+a_{12}+y_{1}=a_{12}+a_{22}+x_{2}$ to obtain a refinement matrix

$$
\begin{array}{lll}
a_{12} & a_{22} & x_{2} \\
a_{11} \\
a_{12} \\
y_{1}
\end{array}\left(\begin{array}{lll}
c_{11} & c_{12} & c_{13} \\
c_{21} & c_{22} & c_{23} \\
c_{31} & c_{32} & c_{33}
\end{array}\right)
$$

In particular, $c_{12} \leq\left\{a_{11}, a_{22}\right\}$, and so we can remove $c_{12}$ from $a_{11}$ and $a_{22}$ as long as we add it to $a_{12}$ and $a_{21}$. More precisely, we obtain a new refinement matrix for the equality $a+a+x=a+a+y$ as follows:

$$
\begin{gathered}
a \\
a \\
a
\end{gathered}\left(\begin{array}{ccc}
c_{11}+c_{13} & c_{12}+a_{12} & y_{1} \\
c_{12}+a_{21} & c_{22}+c_{32} & y_{2} \\
x_{1} & x_{2} & y_{3}
\end{array}\right)
$$

Further, $c_{11}+c_{13} \leq a_{12}+x_{2} \leq c_{12}+a_{12}+x_{2}$ and $c_{22}+c_{32} \leq a_{12}+y_{1} \leq c_{12}+a_{12}+y_{1}$. Hence, after replacing our first refinement matrix with the new one, we may assume that $a_{11} \leq a_{12}+x_{2}$ and $a_{22} \leq a_{12}+y_{1}$. 
With a similar argument, based on a refinement of the equality $a_{11}+a_{21}+x_{1}=a_{21}+$ $a_{22}+y_{2}$, we may assume in addition that $a_{11} \leq a_{21}+y_{2}$ and $a_{22} \leq a_{21}+x_{1}$.

Observe that $x_{1}, x_{2}, y_{1}, y_{2} \in S$. Hence, in $M / S$ we have $\left[a_{11}\right]_{S}+\left[a_{12}\right]_{S}=[a]_{S}=$ $\left[a_{11}\right]_{S}+\left[a_{21}\right]_{S}$, with $\left[a_{11}\right]_{S} \leq\left\{\left[a_{12}\right]_{S},\left[a_{21}\right]_{S}\right\}$. Since $M / S$ is separative, $\left[a_{12}\right]_{S}=\left[a_{21}\right]_{S}$, and thus $a_{12}+t_{1}=a_{21}+t_{2}$ for some $t_{1}, t_{2} \in S$.

Now we have

$$
\begin{aligned}
a+x+t_{1} & =a_{11}+a_{12}+y_{1}+x+t_{1}=a_{11}+a_{21}+y_{1}+x+t_{2} \\
& =a+y_{1}+x_{2}+y_{3}+t_{2}=a_{21}+a_{22}+y+x_{2}+t_{2} \\
& =a_{12}+a_{22}+y+x_{2}+t_{1}=a+y+t_{1}
\end{aligned}
$$

Since $t_{1} \in S$ and $t_{1} \propto a$ (because the o-ideal generated by $a$ is $M$ ), Lemma 4.4 gives us $a+x=a+y$. Finally, note that $a+y=a+x=b+y$ with $y \in S$ and $y \propto a, y \propto b$, so that Lemma 4.4 yields $a=b$ as desired.

\section{StRong SEPARATIVITY}

As indicated in the previous section, there is a strong form of separativity that can hold even when cancellation still fails. The Extension Theorem leads to a corresponding result for strong separativity which allows us to show that the finitely generated projective modules over many exchange rings, including a number of seemingly pathological examples, satisfy strong separativity.

Lemma 5.1. Let $\mathcal{C}$ be a class of modules, closed under finite direct sums. Then the following conditions are equivalent:

(a) For $A, B, C \in \mathcal{C}$, if $A \oplus C \cong B \oplus C$ and $C \lesssim^{\oplus} n A$ for some $n \in \mathbb{N}$, then $A \cong B$.

(b) For $A, B \in \mathcal{C}$, if $2 A \cong A \oplus B$, then $A \cong B$.

(c) For $A, B, C \in \mathcal{C}$, if $A \oplus 2 C \cong B \oplus C$, then $A \oplus C \cong B$.

Proof. Straightforward. (Compare [6, Proposition 4.2].)

We shall say that a class $\mathcal{C}$ of modules, closed under finite direct sums, is strongly separative if the conditions of Lemma 5.1 hold. Condition (a), for finitely generated projective modules, was considered in [6] under the name cancellation of small projectives.

Let us say that a ring $R$ (or an ideal $I$ of $R$ ) is strongly separative provided $F P(R)$ (or $F P(I)$ ) is strongly separative. As with Lemma 4.1, it is clear from Lemma 1.5 that an ideal $I$ of an exchange ring $R$ is strongly separative if and only if the corner rings $e R e$ are strongly separative for all idempotents $e \in I$. Strongly separative exchange rings form a large subclass of separative exchange rings. On the other hand, since members of this subclass have stable rank at most 2 (see Theorem 3.3), there are many examples of separative exchange rings which lie outside this subclass. In fact, there exist separative regular rings with rank functions which are not strongly separative (see [5, Example 3.8]; Theorem 4.2 can be used to show that these examples are separative). The exact connection between separativity and strong separativity will be given in Proposition 5.6.

We can now state our Extension Theorem for strong separativity in exchange rings. This result will follow immediately from Proposition 1.4 and Theorem 5.5. 
Theorem 5.2. Let $R$ be an exchange ring and $I$ an ideal of $R$. Then $R$ is strongly separative if and only if so are $I$ and $R / I$.

As an application of Theorem 5.2 we see that right semiartinian exchange rings are strongly separative. Bergman's example of a directly finite regular ring $R$ which is not unit-regular [25, Example 5.10] is right and left semiartinian; hence, $V(R)$ is strongly separative but not cancellative. Note that [7, Example 3.1] gives an example of a directly finite regular ring which is right semiartinian and a right $\mathrm{V}$-ring (i.e., all simple right modules are injective), but not unit-regular.

The analog of Lemma 5.1 for a monoid $M$ is that the following conditions are equivalent:

(a) For $a, b, c \in M$, if $a+c=b+c$ and $c \propto a$, then $a=b$.

(b) For $a, b \in M$, if $2 a=a+b$, then $a=b$.

(c) For $a, b, c \in M$, if $a+2 c=b+c$, then $a+c=b$.

We say that $M$ is strongly separative provided these conditions are satisfied.

Lemma 5.3. If $S$ is an o-ideal of a strongly separative monoid $M$, then $S$ and $M / S$ are strongly separative.

Proof. Obviously $S$ is strongly separative. Consider $a, b \in M$ such that $2[a]_{S}=[a]_{S}+[b]_{S}$ in $M / S$. Then there exist $e, f \in S$ such that $2 a+e=a+b+f$, that is, $(a+e)+a=(b+f)+a$. Since $a \leq a+e$, it follows from strong separativity in $M$ that $a+e=b+f$. Therefore $[a]_{S}=[b]_{S}$, proving that $M / S$ is strongly separative.

Lemma 5.4. A monoid $M$ is strongly separative if and only if $M$ is separative and all the o-simple factors of principal o-ideals of $M$ are cancellative.

Proof. Any factor of an o-ideal of $M$ is strongly separative by Lemma 5.3. Since an o-simple strongly separative monoid is cancellative, we get one of the implications.

Now assume that $M$ is separative and that all the o-simple factors of all the principal o-ideals of $M$ are cancellative. Let $a, b \in M$ be such that $2 a=a+b$. Denote by $I$ and $J$ the o-ideals generated by $a$ and $b$ respectively. Clearly $J \subseteq I$. If $I=J$ then $a=b$ by separativity of $M$. If $J$ is strictly contained in $I$, then we can choose a maximal proper o-ideal $S$ of $I$ containing $J$, and we obtain that $2[a]_{S}=[a]_{S} \neq[0]_{S}$ in $I / S$, contradicting the assumption that $I / S$ is cancellative. Therefore $a=b$ and $M$ is strongly separative.

Theorem 5.5. Let $M$ be a refinement monoid and $S$ an o-ideal of $M$. Then $M$ is strongly separative if and only if $S$ and $M / S$ are strongly separative.

Proof. One implication is given by Lemma 5.3. Conversely, assume that $S$ and $M / S$ are strongly separative. Then all the o-simple factors of principal o-ideals of $S$ and $M / S$ are cancellative. Now consider an arbitrary o-simple factor $I / J$ of a principal o-ideal $I$ of $M$. If $I \cap S \subseteq J$, then

$$
I / J=I /(I \cap(J+S)) \cong(I+S) /(J+S) \cong((I+S) / S) /((J+S) / S)
$$

with $(I+S) / S$ a principal o-ideal of $M / S$. On the other hand, if $I \cap S \nsubseteq J$, then $(I \cap S)+J=I$ by the maximality of $J$ and so $I / J \cong(I \cap S) /(J \cap S)$; since this monoid has an order-unit, it is isomorphic to a factor of a principal o-ideal of $S$. In either of the above cases, we conclude that $I / J$ is cancellative. Since $M$ is separative by Theorem 4.5 , the result follows from Lemma 5.4 .

We conclude the section with the following ring-theoretic analog of Lemma 5.4. 
Proposition 5.6. An exchange ring $R$ is strongly separative if and only if $R$ is separative and all simple factor rings of corners of $R$ are directly finite, if and only if $R$ is separative and all simple factor rings of corners of $R$ have stable rank 1 .

Proof. This follows from Propositions 1.4, Theorem 3.4, and Lemmas 1.5, 5.4, together with the observation that the principal o-ideals of $V(R)$ are precisely the o-ideals of the form $V(R e R)$ for idempotents $e \in R$. It is clear that $V(R e R)$ is the o-ideal generated by $[e R]$. Conversely, the o-ideal of $V(R)$ generated by a class $[A]$ is easily seen to equal $V(I)$ where $I$ is the trace ideal of $A$. We can write $A \cong e_{1} R \oplus \cdots \oplus e_{n} R$ for some idempotents $e_{i} \in R$, and then $I=R e_{1} R+\cdots+R e_{n} R$. In view of Lemma 1.5, $I=R e R$ for some idempotent $e$, and the proof is complete.

\section{Separative REgular RINGS}

Since regular rings constitute the most thoroughly investigated class of exchange rings, and since many of the cancellation problems to which separativity is related were originally formulated over regular rings, we summarize our main results in this context and discuss their relations with various open questions. In particular, we observe that several basic open problems in this area have positive answers within the class of separative regular rings. We also develop an elementwise characterization of separativity for regular rings, which we use to pinpoint the relationship between separativity and unit-regularity.

Separativity for regular rings is apparently the norm, in that it holds for all known classes of regular rings and is preserved in standard constructions. For instance, the class of separative regular rings includes all unit-regular rings, all right or left $\aleph_{0}$-continuous regular rings (see [1, Theorem 2.13]), and all regular rings satisfying general comparability - in fact, all regular rings satisfying 'generalized s-comparability' [41, Theorem 3.9(2)]. By Proposition 2.2, this class is closed under taking corners, finite matrix rings, arbitrary direct products, direct limits, and factor rings. Further, the class is closed under extensions of ideals by factor rings, by [25, Lemma 1.3] and the Extension Theorem (4.2).

The presence of separativity in a regular ring has a number of nontrivial positive implications, which we summarize in the following theorem. For this reason, separativity was awarded a 'blue ribbon' in [28].

Recall that a ring $R$ is a right (left) Hermite ring $[\mathbf{3 3}]$ provided every $1 \times 2(2 \times 1)$ matrix over $R$ is equivalent to a diagonal matrix. These conditions are equivalent for regular rings [36, Proposition 8]. Further, a regular ring $R$ is Hermite if and only if

$$
2 R \oplus A \cong R \oplus B \quad \Longrightarrow \quad R \oplus A \cong B
$$

for all $A, B \in F P(R)$ [36, Theorem 9].

Theorem 6.1. Let $R$ be a separative regular ring.

(a) If $R$ is directly finite, then $R$ is stably finite.

(b) If $R$ is simple and directly finite, then $R$ is unit-regular.

(c) The stable rank of $R$ is 1,2, or $\infty$.

(d) If $R$ has finite stable rank, then $R$ is a Hermite ring.

(e) Every square matrix over $R$ is equivalent to a diagonal matrix.

Proof. Properties (a)-(d) follow directly from Proposition 2.3 and Theorems 3.3, 3.4. Part (e) is $[4$, Theorem 2.5]. 
Each of the five parts of Theorem 6.1 is itself the subject of an outstanding open problem - namely, does that implication or statement hold universally for regular rings? Parts (a) and (b) correspond to Open Problems 1 and 3 in [25], parts (c) and (d) arose from [36], while part (e) corresponds to Question 6 in [37]. It is generally regarded that, on balance, the first four of these problems (which are seemingly independent) are likely to have negative answers. In this light, it seems rather likely that non-separative regular rings should exist. One of the reasons that current construction techniques have not yielded nonseparative examples is that the class of separative regular rings is closed under extensions. This is in sharp contrast with, say, the class of unit-regular rings. For instance, the first example by Bergman of a directly finite regular ring which is not unit-regular [25, Example 5.10] was constructed as an extension of two unit-regular (in fact, semisimple) rings.

As Theorem 6.1 and the discussion above show, separativity plays a key role in the direct sum decomposition theory of regular rings. Thus the question whether separativity holds universally appears as a fundamental problem, which we emphasize by formulating the

Separativity Problem. Are all regular rings separative?

For a regular ring $R$, cancellativity for $F P(R)$ can be characterized entirely within the ring $R$ by an elementwise property, namely, that each $a \in R$ be unit-regular ( $a=a u a$ for some unit $u$ ). Unit-regularity of certain elements of $R$ also serves to characterize separativity. The characterization is as follows; we write $r(a)$ and $\ell(a)$ for the right and left annihilators of an element $a$.

Proposition 6.2. A regular ring $R$ is separative if and only if each $a \in R$ satisfying

$$
\operatorname{Rr}(a)=\ell(a) R=R(1-a) R
$$

is unit-regular in $R$.

Proof. Firstly assume that $R$ is separative and $a \in R$ satisfies (*). Let $J=R(1-a) R$ and choose an idempotent $g \in J$ such that $1-a \in g R g$. (Such an idempotent exists by [31, Lemma 2.4].) Note that $J=R g R$ and $a=y+(1-g)$ where $y=a g=g a$ is in $g R g$. Also $r(a) \subseteq g R$ and $\ell(a) \subseteq R g$. Let $A=r(a)$ and choose principal right ideals $B$ and $C$ such that $g R=A \oplus C=B \oplus y R$. Then $y R=a g R=a C \cong C$, so $A \oplus C \cong B \oplus C$. Now $g R \subseteq J=R A$ by $\left(^{*}\right)$, whence $g R \lesssim^{\oplus} n A$ for some $n$. Also by the second equality in (*), $g R \subseteq J=\ell(a) R$ and so $g R \lesssim^{\oplus} m(R / a R) \cong m(g R / y R) \cong m B$ for some $m$. Therefore by separative cancellation we can cancel $C$ from $A \oplus C \cong B \oplus C$ to obtain $A \cong B$. Finally, we see that $a$ is unit-regular because $r(a)=A \cong B \cong g R / y R \cong R / a R$.

Conversely, assume $\left(^{*}\right)$ always implies the element $a$ is unit-regular. By Corollary 2.9, it is enough to show that we can obtain cancellation of $C$ in the special case

$$
A \oplus C \cong B \oplus C \lesssim^{\oplus} R
$$

where $A, B, C$ are principal right ideals of $R$ satisfying $C \lesssim A$ and $C \lesssim B$. Write $R=A_{1} \oplus C_{1} \oplus D=B_{1} \oplus C_{2} \oplus D$ where $A_{1} \cong A$ and $B_{1} \cong B$ while $C_{1} \cong C_{2} \cong C$. Let $a \in R$ induce (by left multiplication) an endomorphism of $R_{R}$ which is zero on $A_{1}$, an 
isomorphism from $C_{1}$ onto $C_{2}$, and the identity on $D$. Then $(1-a) R \lesssim^{\oplus} A_{1} \oplus C_{1} \lesssim^{\oplus}$ $2 A_{1}=2 r(a)$, whence $(1-a) R \subseteq \operatorname{Rr}(a)$ and so $R(1-a) R=\operatorname{Rr}(a)$. Also, $R / a R \cong B_{1}$ yields $(1-a) R \lesssim \oplus A_{1} \oplus C_{1} \cong B_{1} \oplus C_{2} \lesssim \oplus 2 B_{1} \cong 2(R / a R)$, and therefore $(1-a) R \subseteq \ell(a) R$. Hence, $R(1-a) R=\ell(a) R$. Now $a$ satisfies $\left(^{*}\right)$ and so, by assumption, $a$ is unit-regular. Thus $r(a) \cong R / a R$ which implies $A \cong A_{1}=r(a) \cong R / a R \cong B_{1} \cong B$ and yields the desired cancellation.

Proposition 6.2 allows us to give the following connection between separativity and unit-regularity, parallel to [6, Proposition 4.9].

Proposition 6.3. A regular ring $R$ is unit-regular if and only if $R$ is separative, every factor ring of $R$ is directly finite, and units can be lifted modulo every ideal of $R$.

Proof. Direct finiteness and separativity are obvious consequences of unit-regularity. That units lift is an old folklore result, recently recorded in [7, Lemma 3.5].

Conversely, assume that the given conditions hold, and let $a \in R$ and $I=\operatorname{Rr}(a)$. In the factor ring $\bar{R}=R / I$, the right annihilator of $\bar{a}$ is zero, and so $\bar{R} \bar{a}=\bar{R}$. By assumption, $\bar{a}$ is a unit of $\bar{R}$ and lifts to a unit $u \in R$. Set $b=u^{-1} a$. Then $I=\operatorname{Rr}(b)$ and $1-b \in I$, whence $R r(b)=R(1-b) R \supseteq \ell(b) R$. Since $R / \ell(b) R$ is directly finite, we obtain that $r(b) \subseteq \ell(b) R$. Thus $R r(b)=\ell(b) R=R(1-b) R$, which by separativity and Proposition 6.2 implies $b$ is unit-regular. Now $b$ equals a unit times an idempotent, whence $a=u b$ has the same form, and so $a$ is unit-regular. Therefore $R$ is unit-regular.

\section{APPLICATIONS TO OPERATOR ALGEBRAS}

The cancellation problems for finitely generated projective modules over regular rings discussed in the previous section all have analogs over $\mathrm{C}^{*}$-algebras, although in that setting it is common to phrase them in terms of orthogonal sums of projections (self-adjoint idempotents). The parallels between the two situations, in terms of what is known and what is open, are particularly striking for $\mathrm{C}^{*}$-algebras whose 'real rank' (see below) is zero. We prove here that these parallels are not just coincidental - the $\mathrm{C}^{*}$-algebras with real rank zero are precisely those $\mathrm{C}^{*}$-algebras which are exchange rings. This theorem then allows our separativity results to be applied to $\mathrm{C}^{*}$-algebras with real rank zero. We summarize the main applications using operator algebra terminology and notation, for the convenience of operator algebraic readers.

We refer the reader to [9] and [26] for background and notation for $\mathrm{C}^{*}$-algebras. In particular, we use $\sim$ and $\lesssim$ to denote Murray-von Neumann equivalence and subequivalence of projections, and we write $M_{\infty}(A)$ for the (non-unital) algebra consisting of $\omega \times \omega$ matrices over an algebra $A$ with only finitely many nonzero entries. Unless specifically noted, our $\mathrm{C}^{*}$-algebras are not assumed to be unital.

The concept of real rank zero for a $\mathrm{C}^{*}$-algebra $A$ has a number of equivalent characterizations (see [14]). The one that relates most naturally to orthogonal sums of projections is the requirement that each self-adjoint element of $A$ can be approximated arbitrarily closely by real linear combinations of orthogonal projections. (This is usually phrased as saying that the set of self-adjoint elements of $A$ with finite spectrum is dense in the set of all self-adjoint elements.) The main result of this section is that the unital $C^{*}$-algebras of real rank zero are exactly the $C^{*}$-algebras which are exchange rings. Since the class of 
$\mathrm{C}^{*}$-algebras of real rank zero is quite large (see for example [11]), this gives a wealth of new examples of exchange rings. In particular, all von Neumann algebras, AF-algebras, irrational rotation algebras and simple purely infinite $\mathrm{C}^{*}$-algebras are exchange rings. (See [11] and the references therein.)

Lemma 7.1. Let $A$ be a unital Banach algebra such that for each $a \in A$ there exists an idempotent $e \in A a$ satisfying $\|1-e\| \leq 1$ and $\|a-a e\|<1$. Then $A$ is an exchange ring.

Proof. Let $a \in A$, and choose an idempotent $1-e \in A(1-a)$ with $\|e\| \leq 1$ such that $\|(1-a)-(1-a)(1-e)\|<1$. Then $\|e-a e\|<1$, and as $\|e\| \leq 1$, we have that $\|e-e a e\|<1$. Thus, eae is invertible in the Banach algebra $e A e$. Let $t \in e A e$ such that $t(e a e)=(e a e) t=e$, and set $g=e+t a(1-e)$. Then, $g=g^{2}$ and also $t a=$ ete $(e a)=e t e(e a e+e a(1-e))=e+t a(1-e)=g$, whence $g \in A a$. On the other hand, $1-g=1-e-t a(1-e)=(1-t a)(1-e) \in A(1-e) \subseteq A(1-a)$. By Lemma 1.1, $A$ is an exchange ring.

Let $A$ be a $C^{*}$-algebra. For $\epsilon>0$, denote by $f_{\epsilon}$ the continuous function from $\mathbb{R}$ to $\mathbb{R}$ which is 0 on $(-\infty, \epsilon / 2]$, linear on $[\epsilon / 2, \epsilon]$, and 1 on $[\epsilon,+\infty)$. For a positive element $x$ in $A$, the set $\left\{f_{\epsilon}(x) \mid \epsilon>0\right\}$ forms an approximate identity for the hereditary sub-C*-algebra generated by $x$, namely $(x A x)^{-}$. As noted in [42, proof of Theorem 7.2], if for each $\epsilon>0$ there is a projection $p_{\epsilon} \in A$ such that $f_{2 \epsilon}(x) \leq p_{\epsilon} \leq f_{\epsilon / 2}(x)$, then the projections $p_{\epsilon}$ form an approximate identity for $(x A x)^{-}$.

Theorem 7.2. Let $A$ be a unital $C^{*}$-algebra. Then the following conditions are equivalent:

(a) $A$ has real rank zero.

(b) $A$ is an exchange ring.

(c) For any positive element $x$ in $A$ and any $\epsilon>0$, there exists a projection $p \in x A x$ such that $f_{\epsilon}(x) \in p A p$.

(d) For each positive element $x \in A$, there exists a projection $p$ in $A$ such that $p \in x A$ and $1-p \in(1-x) A$.

Remark. As the proof shows, it is also equivalent to ask that conditions (c) or (d) hold for all self-adjoint elements, or that condition (d) hold for elements $x$ such that $0 \leq x \leq 1$.

Proof. (a) $\Longrightarrow$ (b). By a result of Menal [35, Proposition 4.8], every unital C*-algebra with real rank zero satisfies the hypothesis of Lemma 7.1.

(b) $\Longrightarrow$ (c). Let $x \geq 0$ in $A$ and $\epsilon>0$. By Lemma 1.1, there exists an idempotent $e \in A$ such that $e \in f_{\epsilon / 2}(x) A$ and $1-e \in\left(1-f_{\epsilon / 2}(x)\right) A$. Observe that $f_{\epsilon / 4}(x) e=e$, so that $g:=e f_{\epsilon / 4}(x)$ is an idempotent and $e A=g A$. Now set $z=1+\left(g^{*}-g\right)\left(g-g^{*}\right)$ and observe that $p=g^{*} g z^{-1}$ is a projection in $A$ (cf. [9, Proposition 4.6.2]). Note that $p A=g^{*} A \subseteq f_{\epsilon / 4}(x) A$ and so $p \in f_{\epsilon / 4}(x) A f_{\epsilon / 4}(x) \subseteq x A x$.

On the other hand, since $1-e \in\left(1-f_{\epsilon / 2}(x)\right) A$, we have $f_{\epsilon}(x)(1-e)=0$ and so $f_{\epsilon}(x)=f_{\epsilon}(x) e$. Consequently

$$
f_{\epsilon}(x) g=f_{\epsilon}(x) e f_{\epsilon / 4}(x)=f_{\epsilon}(x) f_{\epsilon / 4}(x)=f_{\epsilon}(x) .
$$

This implies that $f_{\epsilon}(x) \in g^{*} A=p A$. Thus $p$ is a projection in $x A x$ such that $f_{\epsilon}(x) \in p A p$. 
(c) $\Longrightarrow(\mathrm{d})$. Let $x$ be a positive element in $A$. By (c), there exists a projection $p \in x A x$ such that $f_{1 / 2}(x) \in p A p$. By spectral calculus, $\left(1-f_{1 / 2}(x)\right) A \subseteq(1-x) A$. Also, $1-p=\left(1-f_{1 / 2}(x)\right)(1-p)$, and thus $1-p \in\left(1-f_{1 / 2}(x)\right) A \subseteq(1-x) A$.

$(\mathrm{d}) \Longrightarrow(\mathrm{a})$. It is enough to show that for each positive element $x \in A$, the hereditary sub-C ${ }^{*}$-algebra $(x A x)^{-}$has an approximate identity consisting of projections (use [14, Theorem 2.6(iv)]). For this, it suffices to find, for each $\epsilon>0$, a projection $p$ in $A$ such that $f_{2 \epsilon}(x) \leq p \leq f_{\epsilon / 2}(x)$.

Applying condition (d) to the element $f_{\epsilon}(x)$, we get a projection $p \in f_{\epsilon}(x) A$ such that $1-p \in\left(1-f_{\epsilon}(x)\right) A$. Then $f_{2 \epsilon}(x)(1-p)=0$ and so $f_{2 \epsilon}(x)=f_{2 \epsilon}(x) p$. This gives $f_{2 \epsilon}(x) \leq p$. On the other hand, since $p \in f_{\epsilon}(x) A$, we get $f_{\epsilon / 2}(x)^{1 / 2} p=p$ and so $p \leq f_{\epsilon / 2}(x)$, as desired.

Given a (unital) $\mathrm{C}^{*}$-algebra $A$, all idempotents in matrix algebras $M_{n}(A)$ are equivalent to projections (e.g., [9, Proposition 4.6.2], [26, Proposition 19.1]). Hence, the monoid $V(A)$ may be described as the set of Murray-von Neumann equivalence classes of projections from $M_{\infty}(A)$, with addition induced from orthogonal sums. This description of $V(A)$ is taken as the definition by operator algebraists (cf. [9, Section 5.1]). The same definition is also used when $A$ is not unital, and does not conflict with our usage in that case either. Namely, if $A$ is identified with a closed ideal in its unitification $A^{\sim}$ in the standard manner, the above definition of $V(A)$ in terms of projections yields a monoid isomorphic to the one constructed from the class $F P(A) \subseteq F P\left(A^{\sim}\right)$ as in Section 1.

In view of Theorem 7.2, Corollary 1.3 provides an alternative route to Zhang's Riesz decomposition results for projections in $\mathrm{C}^{*}$-algebras with real rank zero [51, Theorem 3.2]:

Theorem 7.3. Let $p_{1}, p_{2}, q_{1}, q_{2}$ be projections in $M_{\infty}(A)$ where $A$ is a $C^{*}$-algebra with real rank zero. If $p_{1} \oplus p_{2} \sim q_{1} \oplus q_{2}$, there exist orthogonal decompositions $p_{1}=r_{11} \oplus r_{12}$ and $p_{2}=r_{21} \oplus r_{22}$ such that $q_{1} \sim r_{11} \oplus r_{21}$ and $q_{2} \sim r_{12} \oplus r_{22}$.

Proof. After replacing $q_{1}$ and $q_{2}$ by equivalent projections, we may work within the unital $\mathrm{C}^{*}$-algebra $\left(p_{1} \oplus p_{2}\right) M_{\infty}(A)\left(p_{1} \oplus p_{2}\right)$, which has real rank zero by [14, Theorem 2.10, Corollary 2.8]. Thus, without loss of generality, we may assume that $A$ is unital and that $p_{1}, p_{2}, q_{1}, q_{2}$ all lie in $A$. By Theorem 7.2 and Corollary 1.3, $V(A)$ is a refinement monoid. The desired result now follows from the description of $V(A)$ as the monoid of equivalence classes of projections from $M_{\infty}(A)$.

This theorem of course includes Zhang's original Riesz decomposition result [52, Theorem 1.1], namely that $p \lesssim q_{1} \oplus q_{2}$ implies $p=r_{1} \oplus r_{2}$ with $r_{i} \lesssim q_{i}$ for each $i$.

Theorem 7.2 together with Theorem 3.2 yields the following means of calculating stable ranks (see the end of Section 3 for the definition of stable rank of elements of a monoid):

Theorem 7.4. If $A$ is a unital $C^{*}$-algebra with real rank zero, then its stable rank equals the stable rank of the element $\left[1_{A}\right]$ in the monoid $V(A)$.

Since the monoid $V(A)$ has the same description in terms of projections in both the unital and non-unital cases, the definition of separativity for $\mathrm{C}^{*}$-algebras can be given in both cases simultaneously. Thus, a $\mathrm{C}^{*}$-algebra $A$ is separative provided that

$$
p \oplus p \sim p \oplus q \sim q \oplus q \quad \Longrightarrow \quad p \sim q
$$


for projections $p, q \in M_{\infty}(A)$. (Some equivalent formulations follow from Lemma 2.1.)

The class of separative $C^{*}$-algebras includes those with stable rank 1 (over which orthogonal sums of projections enjoy cancellation) as well as those whose projections satisfy the condition $p \oplus p \sim q \oplus q \Longrightarrow p \sim q$. Thus, for example, all $\mathrm{AW}^{*}$-algebras, Rickart $\mathrm{C}^{*}$ algebras, AF-algebras, and irrational rotation algebras are separative. It follows from results of Cuntz [20, Theorem 1.4, Proposition 1.5] that all purely infinite simple $C^{*}$-algebras are separative. In work in progress, Brown and Pedersen have shown that $\mathrm{C}^{*}$-algebras of real rank zero which are extremally rich in the sense of $[\mathbf{1 5}]$ are separative (cf. $[\mathbf{1 3}$, Section 1]).

Theorem 7.5. Let $A$ be a $C^{*}$-algebra with real rank zero and assume that $I$ is a closed ideal of $A$. Then $A$ is separative if and only if $I$ and $A / I$ are separative. In particular, $A$ is separative if and only if its unitification is separative.

Proof. This follows from Theorems 4.2 and 7.2 .

We conclude by summarizing our main applications of separativity in the operator algebra context. Recall that a unital $\mathrm{C}^{*}$-algebra $A$ is said to be finite if $x x^{*}=1$ implies $x^{*} x=1$ for $x \in A$; this is equivalent to $A$ being directly finite $[9,6.3 .2]$.

Theorem 7.6. Let $A$ be a unital $C^{*}$-algebra with real rank zero, and assume that $A$ is separative.

(a) If $A$ is finite, then $A$ is stably finite.

(b) If $A$ is simple and finite, then $A$ has stable rank 1 .

(c) The stable rank of $A$ is 1,2 , or $\infty$.

(d) The stable rank of $A$ is finite if and only if the following cancellation property holds for projections $p, q \in M_{\infty}(A)$ :

$$
1_{A} \oplus 1_{A} \oplus p \sim 1_{A} \oplus q \quad \Longrightarrow \quad 1_{A} \oplus p \sim q .
$$

Proof. Because of Theorem 7.2, we can apply Proposition 2.3 and Theorems 3.3, 3.4.

There exist examples of finite unital $\mathrm{C}^{*}$-algebras which are not stably finite. These examples are constructed as extensions of a commutative $\mathrm{C}^{*}$-algebra by the algebra of compact operators on a separable infinite-dimensional Hilbert space $[\mathbf{9}, 6.10 .1]$. By Theorems 7.5 and 7.6 , no such construction gives a finite but not stably finite $\mathrm{C}^{*}$-algebra of real rank zero.

\section{REFERENCES}

[1] P. Ara, Aleph-nought-continuous regular rings, J. Algebra 109 (1987), 115-126.

[2] - Left and right projections are equivalent in Rickart $C^{*}$-algebras, J. Algebra 120 (1989), $433-448$

[3] - Strongly $\pi$-regular rings have stable range one, Proc. Amer. Math. Soc. (to appear).

[4] P. Ara, K.R. Goodearl, K.C. O'Meara, and E. Pardo, Diagonalization of matrices over regular rings (to appear)

[5] P. Ara, K.R. Goodearl, E. Pardo, and D.V. Tyukavkin, K-theoretically simple von Neumann regular rings, J. Algebra 174 (1995), 659-677.

[6] P. Ara, K.C. O'Meara and D.V. Tyukavkin, Cancellation of projective modules over regular rings with comparability, J. Pure Appl. Algebra 107 (1996), 19-38. 
[7] G. Baccella, Semiartinian $V$-rings and semiartinian von Neumann regular rings, J. Algebra 173 (1995), 587-612.

[8] G. Bergman, Personal correspondence, 1990.

[9] B. Blackadar, K-Theory for Operator Algebras, MSRI Publications 5, Springer-Verlag, New York, 1986.

[10] _ Rational $C^{*}$-algebras and non-stable K-theory, Rocky Mountain J. Math. 20 (1990), 285316.

[11] $\longrightarrow$ Projections in $C^{*}$-algebras, in $C^{*}$-Algebras: 1943-1993 (R. S. Doran, Ed.), Contemp. Math. 167 (1994), 131-149.

[12] B. Blackadar and D. Handelman, Dimension functions and traces on $C^{*}$-algebras, J. Func. Anal. 45 (1982), 297-340.

[13] L. G. Brown, Homotopy of projections in $C^{*}$-algebras of stable rank one, in Recent Advances in Operator Algebras (Orléans, 1992), Astérisque 232 (1995), 115-120.

[14] L.G. Brown and G.K Pedersen, $C^{*}$-algebras of real rank zero, J. Func. Anal. 99 (1991), 131-149.

[15] - On the geometry of the unit ball of a $C^{*}$-algebra, J. reine angew. Math. 469 (1995), $113-147$.

[16] V.P. Camillo and H.-P. Yu, Stable range one for rings with many idempotents, Trans. Amer. Math. Soc. 347 (1995), 3141-3147.

[17] A.H. Clifford and G.B. Preston, The algebraic theory of semigroups, Vol. 1, Math. Surveys 7 , Amer. Math. Soc., Providence, 1961.

[18] P.M. Cohn, Algebra, Vol. 1, Second Ed., Wiley, New York, 1982.

[19] P. Crawley and B. Jónssọ, Refinements for infinite direct decompositions of algebraic systems, Pacific J. Math. 14 (1964), 797-855.

[20] J. Cuntz, K-theory for certain $C^{*}$-algebras, Annals of Math. 113 (1981), 181-197.

[21] H. Dobbertin, Refinement monoids, Vaught monoids, and Boolean algebras, Math. Annalen 265 (1983), 473-487.

[22] E. G. Evans, Jr., Krull-Schmidt and cancellation over local rings, Pacific J. Math. 46 (1973), $115-121$.

[23] L. Fuchs, Partially Ordered Algebraic Systems, Pergamon Press, Oxford, 1963.

[24] K. R. Goodearl, Direct sum properties of quasi-injective modules, Bull. Amer. Math. Soc. 82 (1976), 108-110.

[25] - Von Neumann Regular Rings, Pitman, London, 1979; Second Ed., Krieger, Malabar, Fl., 1991.

[26] - Notes on real and complex $C^{*}$-algebras, Shiva Math. Series 5, Nantwich (Cheshire), 1982.

[27] Torsion in $K_{0}$ of unit-regular rings, Proc. Edinburgh Math. Soc. 38 (1995), 331-341.

[28] - Von Neumann regular rings and direct sum decomposition problems, in Abelian Groups and Modules, Padova 1994 (A. Facchini and C. Menini, eds.), Kluwer, Dordrecht, 1995, pp. 249-255.

[29] K.R. Goodearl and R.B. Warfield, Jr., Algebras over zero-dimensional rings, Math. Annalen 223 (1976), 157-168.

[30] P. A. Grillet, Interpolation properties and tensor products of semigroups, Semigroup Forum 1 (1970), 162-168.

[31] J. Hannah and K.C. O'Meara, Products of idempotents in regular rings, II, J. Algebra 123 (1989), $223-239$.

[32] E. Hewitt and H.S. Zuckerman, The $\ell_{1}$-algebra of a commutative semigroup, Trans. Amer. Math. Soc. 83 (1956), 70-97.

[33] I. Kaplansky, Elementary divisors and modules, Trans. Amer. Math. Soc. 66 (1949), 464-491.

[34] N. Kimura and Y.-S. Tsai, On power cancellative archimedean semigroups, Proc. Japan Acad. 48 (1972), 553-554.

[35] P. Menal, Spectral Banach algebras of bounded index, J. Algebra 154 (1993), 27-66.

[36] P. Menal and J. Moncasi, On regular rings with stable range 2, J. Pure Appl. Algebra 24 (1982), 25-40.

[37] J. Moncasi, Rang estable en anells regulars, Ph.D. Thesis, Universitat Autònoma de Barcelona, 1984.

[38] - A regular ring whose $K_{0}$ is not a Riesz group, Communic. in Algebra 13 (1985), $125-131$.

[39] W.K. Nicholson, Lifting idempotents and exchange rings, Trans. Amer. Math. Soc. 229 (1977), 269-278. 
[40] E. Pardo, Monoides de refinament $i$ anells d'intercanvi, Ph.D. Thesis, Universitat Autònoma de Barcelonà, 1995.

[41] - Comparability, separativity and exchange rings, Communic. in Algebra (to appear).

[42] M. Rørdam, On the structure of simple $C^{*}$-algebras tensored with a UHF-algebra, II, J. Func. Anal. 107 (1992), 255-269.

[43] J. Stock, On rings whose projective modules have the exchange property, J. Algebra 103 (1986), $437-453$.

[44] L.N. Vaserstein, Stable rank of rings and dimensionality of topological spaces, Func. Anal. Applic. 5 (1971), 102-110.

[45] R.B. Warfield, Jr., Exchange rings and decompositions of modules, Math. Ann. 199 (1972), 31-36.

[46] - Cancellation of modules and groups and stable range of endomorphism rings, Pacific J. Math. 91 (1980), 457-485.

[47] F. Wehrung, Injective positively ordered monoids I,II, J. Pure Appl. Algebra 83 (1992), 43-82, 83-100.

[48] - Embedding simple commutative monoids into simple refinement monoids, Semigroup Forum (to appear).

[49] T. Wu and W. Tong, Finitely generated projective modules over exchange rings, manuscripta math. 86 (1995), 149-157.

[50] H.-P. Yu, Stable range one for exchange rings, J. Pure Appl. Algebra 98 (1995), 105-109.

[51] S. Zhang, Diagonalizing projections in multiplier algebras and in matrices over a $C^{*}$-algebra, Pacific J. Math. 145 (1990), 181-200.

[52] — A Riesz decomposition property and ideal structure of multiplier algebras, J. Operator Theory 24 (1990), 204-225.

[53] - Certain $C^{*}$-algebras with real rank zero and their corona and multiplier algebras. Part $I$, Pacific J. Math. 155 (1992), 169-197.

P. Ara: Departament de Matemàtiques, Universitat Autònoma de Barcelona, 08193 Bellaterra (BARCELona), Spain

E-mail address: para@mat.uab.es

K.R. Goodearl: Department of Mathematics, University of California, Santa Barbara, CALIFORNIA 93106, USA

E-mail address: goodearl@math.ucsb.edu

K.C. O'Meara: Department of Mathematics, University of Canterbury, Christchurch, NEW ZEALAND

E-mail address: komeara@math.canterbury.ac.nz

E. Pardo: Departament de Matemàtiques, Universitat Autònoma de Barcelona, 08193 Bellaterra (Barcelona), Spain

E-mail address: epardo@mat.uab.es 